\title{
EFECTO DE LA NATACIÓN SOBRE PARÁMETROS FISIOLÓGICOS Y HEMATOLÓGICOS EN EL CABALLO
}

\author{
Effect of swimming on physiological and hematological parameters in \\ the horse
}

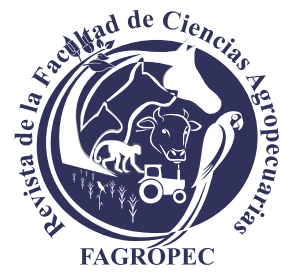

Recibido 20 de julio de 2020 Aceptado 10 de noviembre 2020

'MSc. Universidad Francisco de Paula Santander, GI@SDy GIPAB. Vía Acolsure, Sede el Algodonal, Ocaña, Colombia. E-mail: jfhoyosp@ufpso.edu.co

iD https://orcid.org/0000-0002-0377-4664

${ }^{2}$ MVZ. Universidad de Pamplona, GIAS, Pamplona, Colombia.E-mail: equinoseliecer@hotmail.com

iD https://orcid.org/0000-0003-4035-720X

${ }^{3}$ MSc. Universidad Francisco de Paula Santander, GI@SD y GIPAB, Ocaña, Colombia. E-mail: dahernandezv@ufpso.edu.co

D https://orcid.org/0000-0003-1971-8365

${ }^{4}$ Zootec. Universidad de Pamplona, Colombia.

E-mail: sergiovargas744@hotmail.com

iD https://orcid.org/0000-0002-8189-758X

${ }^{5}$ Universidad de Santander UDES, Facultad de Ciencias de la Salud, Estudiante del programa de Bacteriología y laboratorio Clínico. E-mail: sayagotefi2903@gmail.com

iD https://orcid.org/0000-0002-8440-9510

\section{Cómo citar}

Hoyos Patiño, J. F., Franco Roa, E., Hernández Villamizar, D. A., Vargas Gómez, S. A., \& Sayago Velásquez, J. E. (2020). Efecto de la natación sobre parámetros fisiológicos y hematológicos en el caballo. Revista Facultad de Ciencias Agropecuarias -FAGROPEC. Universidad de la Amazonia. Vol. 12(2)202-209. https://doi.org/10.47847/fagropec.v12n2a3

*Autor para correspondencia: jfhoyosp@ufpso.edu.co

Este artículo puede compartirse bajo la Licencia Creative Commons (CC BY 4.0).

\section{Johann Fernando Hoyos Patiño ${ }^{1 *}$ Eliecer Franco Roa $^{2}$ Daniel Antonio Hernández Villamizar ${ }^{3}$ Sergio Alberto Vargas Gómez ${ }^{4}$ Jeimy Estefanny Sayago Velásquez ${ }^{5}$}

\section{RESUMEN}

El presente estudio describe la frecuencia cardíaca, la frecuencia respiratoria y los parámetros hematológicos de caballos criollos colombianos entrenados en piscina, en el criadero Villa María ubicado en la vereda El Palmar de Villa del Rosario, en el Norte de Santander. Se realizó seguimiento de 7 equinos criollos Colombianos, de entre 36 a 60 meses de edad, tomando muestras en reposo (T0), al momento de terminar el entrenamiento (T1) y 15 minutos después de terminar el entrenamiento (T2). El enfoque de la investigación fue cuantitativo, fundamentado en la medición de las características del fenómeno, delimitado por las variaciones de las constantes fisiológicas y hematológicas de los equinos evaluados. El análisis de los resultados demostró que los muestreos T0 y T2 presentaron parámetros fisiológicos dentro de los rangos normales, tanto para frecuencia cardiaca, como para frecuencia respiratoria y parámetros hematológicos. En el muestreo T1 los linfocitos y monocitos mostraron diferencia en relación con los otros muestreos, donde los linfocitos descendieron y los monocitos superaron el nivel normal, lo cual es indicativo de un mayor grado de respuesta al estrés.

Palabras clave

Caballo colombiano; natación; parámetros, fisiológicos; hematológicos.

\section{ABSTRACT}

The present study describes the heart rate, respiratory rate and hematological parameters of Colombian Criollo horses trained in a swimming pool at the Villa María breeding farm in El Palmar, Villa del Rosario in the North of Santander. Seven Colombian Criollo horses between 36 and 60 months old were sampled at rest (T0), at the end of the training (T1) and 15 minutes after finishing the training (T2). The focus of this research was quantitative, based on measuring the characteristics of the phenomenon, delimited by the variations of physiological and hematological constants of the evaluated equines. The analysis of the results showed that the T0 and T2 samples presented physiological parameters within the normal ranges for heart rate, respiratory rate and hematological parameters. In the T1 sampling, the lymphocytes and monocytes showed difference in relation to the other samples, where the lymphocytes decreased and the monocytes exceeded the normal level. These results indicated a higher degree of response to stress.

Key words

Colombian horse; hematological; physiological; swimming. 


\section{INTRODUCCIÓN}

La natación en piscina, como alternativa de entrenamiento para caballos de alto rendimiento, desarrolla fortaleza y potencializa las cualidades del equino (Corvalán, 2000; Hoyos y Franco, 2020a). Esta actividad también es recomendada para la recuperación física por lesiones músculoesqueléticas, pues evita la atrofia muscular producto del reposo, acortando el proceso de rehabilitación(Davie, etal., 2008).

La natación es utilizada como entrenamiento cruzado, apoyando el entrenamiento cardiovascular para el equino y presenta la ventaja de reducir al mínimo la tensión diaria en las articulaciones y las extremidades, favoreciendo su flexibilidad (Hobo, et al., 1998; Hoyos y Franco 2020b). Por otra parte la natación se puede considerar, como un ejercicio no específico del entrenamiento, que permite excelentes resultados, con la aplicación gradual durante el proceso de doma y adiestramiento (Corvalán, 2000).

El adiestramiento del caballo criollo colombiano, entendido como un proceso de intervención antrópica, tiene como fin hacer que el ejemplar desarrolle, con ayuda de un régimen de entrenamiento progresivo, todo su potencial físico. El éxito o fracaso del proceso determina en gran parte el rendimiento en pista, por lo que un animal adiestrado inadecuadamente, probablemente no pueda desempeñarse satisfactoriamente en competencia, llegando en la mayoría de los casos, a considerarse su descarte (Hoyos, 2016).

La alternativa de la natación en piscina para potenciar el proceso de entrenamiento de los caballos es muy amplia, brindando un ambiente progresivo para las necesidades cardiovasculares, minimizando así el impacto en el sistema musculoesquelético (Hoyos, 2020). Por tanto, estudiar el efecto de su aplicación sobre las constantes fisiológicas y hematológicas, determinará el nivel de bienestar animal, cuando el ejemplar sea sometido a este proceso. (Patiño, et al, 2019).

Teniendo en cuenta lo expuesto anteriormente surge la pregunta ¿cuáles son los efectos fisiológicos y hematológicos de la aplicación del entrenamiento de natación en piscina, en el caballo de silla colombiano?

En tal sentido, el objetivo general de esta investigación, es el de describir el efecto del entrenamiento de natación en piscina, sobre la frecuencia cardiaca, la frecuencia respiratoria y los parámetros hematológicos, en caballos criollos colombianos del criadero Villa María de la ciudad de Cúcuta.

A su vez, los objetivos específicos planteados fueron los siguientes:

- Caracterizar el proceso de entrenamiento de natación en piscina, aplicado a los equinos de competencia del criadero Villa María.

- Determinar la variación de la frecuencia cardiaca y de las frecuencias respiratorias pre y post entrenamiento de natación en piscina, en caballos criollos colombianos.

- Establecer los cambios sucedidos de las constantes hematológicas pre y post entrenamiento de natación en piscina, en caballos criollos colombianos. 


\section{METODOLOGÍA}

El enfoque de la investigación fue cuantitativo. Según (Taylor y Bogdan, 2004), el cual se fundamenta en la medición de las características del fenómeno. En este caso, delimitando por las variaciones de las constantes fisiológica y hematológicas de los equinos evaluados. El tipo de investigación aplicada fue descriptiva, ya que reseña las características de un fenómeno existente, a través de la recolección de datos, interpretación y análisis, en atención al universo real de donde proviene (Arias, 2006), caracterizando el comportamiento de las constantes fisiológicas y hematológicas durante el periodo de entrenamiento. La selección de los ejemplares se amparó en el tipo de muestra no probabilística por conveniencia (Balestrini, 2006), el cual permite seleccionar los ejemplares objeto de estudio, dadas las características de disponibilidad de acceso, de colaboración del criador y de presupuesto para la ejecución de la investigación(Bernal, 2016).

\section{RESULTADOS Y DISCUSIÓN}

Área de estudio. Se llevó a cabo en el Criadero Villa María, en Villa del Rosario al Norte de Santander; a una altitud de $440 \mathrm{msnm}$. coordenadas satelitales latitud 7.8566226 longitud 72.45360370000003 , con temperatura promedio de $28^{\circ}$ y máxima de $35^{\circ} \mathrm{C}$. El criadero cuenta con una extensión total de 22 hectáreas, de las cuales, 3 hectáreas están destinadas a instalaciones; 2 hectáreas están destinadas para pasto de corte, 10 hectáreas están destinadas para potreros y 7 hectáreas están destinadas para producción de heno. El criadero se encuentra ubicado a una distancia de 25 kilómetros de la ciudad de Cúcuta (Alcaldía de Villa del Rosario, 2019).

Especímenes de estudio. Para este estudio se evaluó un grupo de 7 caballos criollos colombianos de la trocha y el galope, con edades que oscilan entre los 36 a los 60 meses. Los ejemplares fueron evaluados clínica y etológicamente, para evitar ejemplares con alteraciones sanitarias que afectaran la efectividad de los datos.

Levantamiento de la información parámetros fisiológicos. Las variables fisiológicas de frecuencia cardiaca (FC), frecuencia respiratoria (FR), tomadas en los tiempos T0: ejemplar en reposo; T1: después del ejercicio y T2: 15 minutos después del ejercicio.

Frecuencia cardiaca. Fue identificada medio de auscultación con estetoscopio; el campo de auscultación del equino se encuentra delimitado cranealmente por el margen caudal de la escápula y la musculatura del hombro, dorsalmente por la musculatura del lomo, caudalmente se extiende hasta la altura de la tuberosidad coxal y ventralmente hacia el codo (Guerrero et al., 2009).

Frecuencia respiratoria. Fue registrada mediante observación de los movimientos del tórax, de los flancos o de los ollares. También se realizó acercándose al caballo, colocando la mano en los ollares, para notar la salida de aire y contar las respiraciones por minuto. Para la auscultación directa con fonendoscopio, se ubicó la campana de auscultación en los primeros anillos traqueales a la altura de la laringe, que se pueden palpar en la zona ventral del cuello. En un caballo sano es de 8-20 rpm (en los potros 20-30 rpm) (Guerrero et al., 2009). 
Muestra sanguínea. La muestra de sangre se tomó con aguja calibre 16, 18 o 21 en la vena yugular, previa desinfección de la zona; se extrajo un total de $5 \mathrm{ml}$ de sangre por animal, el cual fue depositado en tubo con solución anticoagulante de EDTA (Vacutainner tapa lila), este fue completamente rotulado, homogenizado suavemente y refrigerado para su envío al laboratorio, donde se analizó con la máquina Prenta Ochenta (Patiño-Quiroz et al., 2016).

Análisis estadístico. Se propone que todos los participantes del estudio sean tratados como una unidad experimental para el desarrollo de la investigación. Para el análisis de datos, cada muestra se estima como una variable de respuesta para el método de procesamiento y las constantes fisiológicas y sanguíneas obtenidas.

Los datos se presentan como promedio y desviación estándar, calculados para cada una de las variables analizadas, con el fin de presentar datos descriptivos sobre el comportamiento de los parámetros.

Implicaciones éticas. Según Estrada-Cely y Parra-Herrera (2016), la bioética puede expresarse desde una postura deontológica o microbioética, que asume y analiza las interacciones al interior de las relaciones, entre los individuos de la especie humana, entre las personas, y se fundamenta en el principio de igualdad, establecido desde el respeto a la autonomía y desde una conducta no maliciosa. La otra forma de expresión de la bioética es la macrobioética desde la que se asume y se analizan las interacciones entre individuos de diferentes especies, fundamentándose en la desigualdad, desde el que se entiende que el ser humano posee un estado de superioridad, que supone también mayor responsabilidad, en razón a su condición como uno ser con sentido moral; se entiende entonces desde esta postura, que la toma de decisiones en este campo, se encuentra orientada por la justicia en términos de igual consideración con los intereses del otro, considerándolo necesariamente como un ser diferente del humano; igualmente se tiene en cuenta la idea de la compasión, entendida como la continua disposición de ponerse en el lugar del otro (Engelhardt, 1995; Beauchamp y Childress , 1999; Carmona, et al., 1999; Cely, 2002; Beorlegui, 2005; Capó-Marti, 2005; Hottois, 2007).

El conjunto de pautas anteriores, incluyendo los lineamientos específicos establecidos internacionalmente por Cardozo-Martínez (2007), fundamentan los pilares de análisis del Comité de Bioética de la Universidad Francisco de Paula Santander Ocaña, los cuales avalaron el desarrollo de la presente investigación como se indica en la Resolución 043 del 23 de agosto de 2018.

Descripción del comportamiento de la frecuencia cardiaca, frecuencia respiratoria y parámetros hematológicos. Los equinos al momento del estudio, ya tienen entrenamiento previo en piscina y están habituados al protocolo del proceso. Los datos obtenidos se clasificaron en tres momentos de muestreo T0, T1 y T2 (Tabla 1). Cada ejemplar dio 6 vueltas, 3 por cada lado, equivalentes a $1.800 \mathrm{~m}$, para un total de $3.600 \mathrm{~m}$.

Luego de analizar los datos, se observa que existe una diferencia entre los resultados de T0 y T1, al igual que entre los de T1 y T2. Esto se debe al esfuerzo que los caballos realizan durante el proceso de adiestramiento, lo que genera presión en el sistema, lo que se manifiesta como un aumento de los 
Tabla 1.

Frecuencia cardiaca, frecuencia respiratoria y parámetros hematológicos en los tres momentos de muestreo.

\begin{tabular}{lccc}
\hline Variable & $\begin{array}{c}\text { To } \\
\text { Media }\end{array}$ & $\begin{array}{c}\text { T1 } \\
\text { Media }\end{array}$ & $\begin{array}{c}\text { T2 } \\
\text { Media }\end{array}$ \\
\hline FC & $36.33 \pm 13.77$ & $42.02 \pm 1.07$ & $37.02 \pm 8.93$ \\
FR & $17.38 \pm 7.09$ & $24.31 \pm 6.95$ & $19.31 \pm 8.13$ \\
RecL & $10.13 \pm 1.86$ & $11.15 \pm 2.17$ & $10.39 \pm 1.84$ \\
Neut\% & $19.25 \pm 5.74$ & $21.99 \pm 10.86$ & $15.83 \pm 8.13$ \\
Linf\% & $58.80 \pm 10.20$ & $40.72 \pm 10.20$ & $58.80 \pm 6.54$ \\
Mono\% & $9.75 \pm 3.15$ & $17.92 \pm 4.97$ & $9.00 \pm 10.67$ \\
Eosi\% & $2.19 \pm 1.08$ & $2.60 \pm 1.34$ & $1.93 \pm 1.15$ \\
Baso\% & $0.37 \pm 0.31$ & $0.66 \pm 0.33$ & $0.39 \pm 0.69$ \\
Conteritro & $8.68 \pm 1.51$ & $9.11 \pm 2.24$ & $8.61 \pm 1.16$ \\
hemog & $13.47 \pm 2.35$ & $13.88 \pm 2.16$ & $12.82 \pm 1.59$ \\
hemato & $36.81 \pm 5.86$ & $37.66 \pm 5.57$ & $35.46 \pm 4.08$ \\
\hline
\end{tabular}

parámetros estudiados (Tabla 1). Por otro lado, no hay diferencia entre los resultados de las muestras T0 y T2, lo cual se debe al proceso de entrenamiento gradual y a la capacidad de las muestras para volver a un estado estable. (Figura 1).

Además de ello, no hubo una diferencia significativa en la frecuencia cardíaca y respiratoria entre las muestras T0 y T2, que fue superior al rango reportado para esta especie (36-40 p / my 8-20 rpm) (Gutiérrez y Gonzales, 1998; Ramírez, 2005) y Guerrero et al., 2009) (Tabla 1). Sin embargo, debido al esfuerzo cardiovascular que representa la natación, han cambiado en el muestreo T1, con incrementos promedio en la frecuencia cardiaca y respiratoria de $5.69 \mathrm{p} / \mathrm{m}$ y $6.93 \mathrm{rpm}$, respectivamente (Figura 1).

De acuerdo con la evaluación de Castillo et al. (2010), los parámetros hematológicos evaluados no cambiaron significativamente las muestras T0 y T2 (Tabla 1) y aún se encontraban entre los estándares normales para caballos. Además de los porcentajes de linfocitos y monocitos, estos cambiaron durante el muestreo de T1 (Figura 1).

Los linfocitos, que cumplen la función como segunda línea de defensa, después de los neutrófilos, presentaron un descenso de 18.8 puntos porcentuales en el muestreo T1 (Figura 1), ubicándose dentro del rango normal, pero disminuido en relación con los otros dos muestreos. Según García et al. (1995), disminuyeron frente a condiciones de estrés crónico, crecimiento, lesiones, o en caballos con carácter nervioso (Tabla 1).

Los monocitos presentaron un incremento de 8.17 puntos porcentuales en el muestreo T1 (Tabla 1), superando los límites normales (Benjamin, 1991), por lo que representan un parámetro para la determinación de estrés, producto del esfuerzo que realiza el ejemplar en el proceso de natación (Figura 1). 
Figura 1.

Frecuencia cardiaca, frecuencia respiratoria y parámetros hematológicos en caballos criollos colombianos entrenados con natación en piscina.

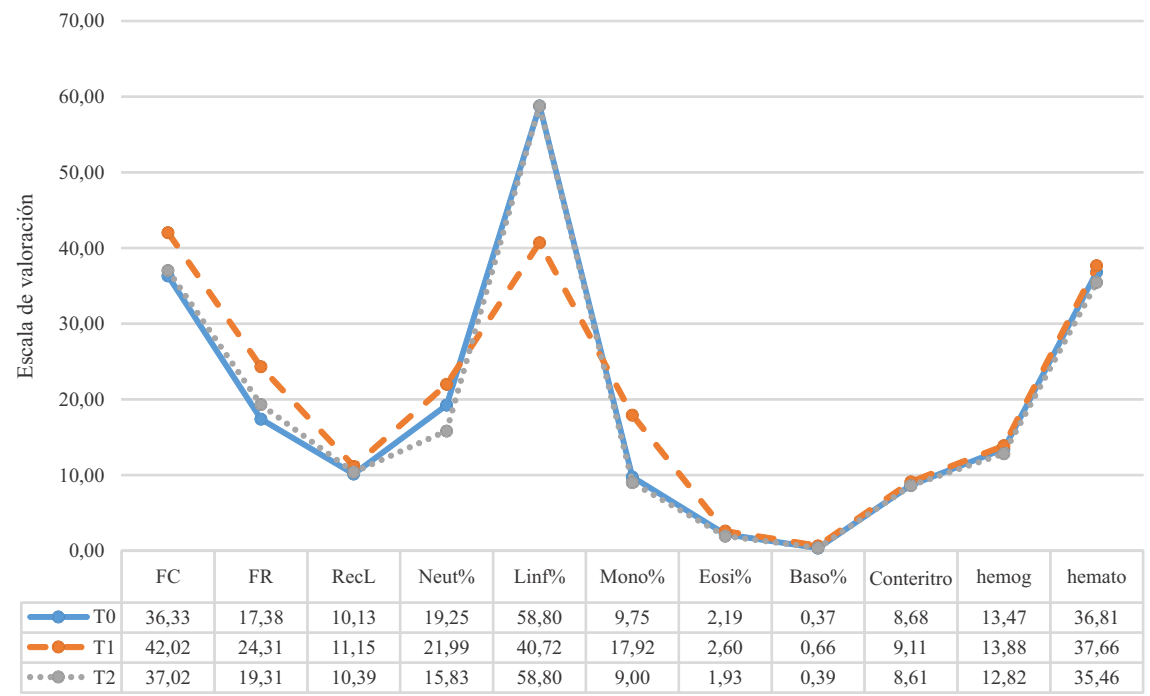

El comportamiento de los ejemplares en el muestreo T2 (Tabla 1), demuestra la capacidad de recuperación, producto del modelo de entrenamiento gradual y de los cuidados por parte del equipo de trabajo, retomando parámetros normales en todas las variables evaluadas (Gutiérrez y González, 1998; Ramírez, 2005 y Guerrero et al., 2009) (Figura 1).

\section{CONCLUSIONES}

Durante el desarrollo del proceso se caracterizó el entrenamiento de natación en piscina, implementado en el criadero Villa María, determinándose la importancia de aplicarlo gradualmente, para lograr el acondicionamiento físico de los ejemplares de alta competencia, en tanto fortalece la capacidad cardiovascular, sin atentar contra el sistema músculo esquelético.

En los muestreos T0 y T2 se presentaron parámetros fisiológicos dentro de los rangos normales para frecuencia cardiaca, frecuencia respiratoria y parámetros hematológicos. En el muestreo T1, los linfocitos y los monocitos mostraron diferencia en relación con los otros muestreos; los linfocitos descendieron y los monocitos superaron el nivel normal, lo cual es indicativo de mayor grado de respuesta a estrés.

Finalmente, de manera general es posible concluir que la natación en piscina como ejercicio no específico en el entrenamiento del caballo criollo colombiano, brinda alternativas para el desarrollo del equino. Sin embargo, este debe ser aplicado de forma gradual, ya que el impacto causado puede llegar a implicar niveles de estrés importantes, si los ejemplares no cuentan con la preparación adecuada y el manejo oportuno de personal idóneo, durante el proceso de aplicación. 


\section{REFERENCIAS BIBLIOGRÁFICAS}

Alcaldía de Villa del Rosario. (2019). Unidos por Villa del Rosario. Sitio oficial del municipio de Villa del Rosario; creado por Gobierno Digital. https://bit.ly/2mlisNi

Arias, F. (2006). El proyecto de investigación introducción a la metodología científica, 5 ta edición. Episteme.

Balestrini, M. (2006). Cómo se elabora el Proyecto de Investigación. Editorial BL Consultores Asociados. Caracas. Venezuela.

Beauchamp, Ly Childress, F. (1999) Principios de Ética Biomédica. España: Masson. S.A,. 522 p.

Benjamin,M. (1991). Manual de Patología Clínica en Veterinaria. Ed. Limusa. México. P 120

Beorlegui, C. (2005) Ética animal e idea de persona. [En línea] Madrid: Universidad Pontifica Comillas.p 14.http://goo.gl/uTTzGZ.

Bernal, C. (2016). Metodología de la investigación. Bogotá: Pearson.

Capó-Marti, M. (2005). Aplicación de la bioética al bienestar y los derechos de los animales. España: Complutense SA, 112 p.

Cardozo-Martinez, CA. (2007). El animal como sujeto experimental, aspectos técnicos y éticos. Chile: CIEB, Universidad de Chile. https://cutt.ly/lgD9Tvx

Castillo, C.; Cano, C.; Suárez, A. (2010). Valores hematológicos en caballos criollos colombianos del Valle de Aburrá. Corporación Universitaria Lasallista. [Fecha de consulta: 12 de mayo de 2016.] http://goo.gl/3XNhrk

Carmona, J., et al. (1999) Macrobioética En: Colección Bios y Ethos. Bogotá: Kimpres. Universidad el Bosque, $1999.13 \mathrm{p}$.

Cely, G. (2002). El horizonte bioético de las ciencias: Reflexiones para elaborar una ecoética. Bogotá: Fundación Cultural Javeriana. Pp. 89-91

Corvalán. RC. (2000) Entrenamiento del caballo de carreras. Real Academia de Ciencias Veterinarias de España. Madrid. https://eprints.ucm.es/11592/1/T32259.pdf

Davie A, CJ. Savage, LF. (2008). Efecto de la natación en las dimensiones cardiacas en caballos PSI. Rural Industries Research and Development Corporation Publication.

Engelhardt, H. (1995). Los fundamentos de la Bioética. Barcelona: Paidos. 545 p.

Estrada-Cely, G. E., \& Parra-Herrera, J. P. (2016). Las implicaciones éticas y bioéticas en la investigación científica. CES Medicina Veterinaria y Zootecnia, 11 (2), 115 - 118. 


\section{https://cutt.ly/GhpwlUA}

García, A., Castejón, M., De la Cruz, P., González, G., López, M., Ruiz, S,. (1995). Fisiología Veterinaria. España. Interamericana. Mc Graw - Hill.1074p.

Guerrero P, Portocarrero L, Mutis C, Ramirez J. (2009). Determinación de frecuencia cardiaca, frecuencia respiratoria, lactato deshidrogenasa, creatinkinasa y ácido láctico en caballos durante competencia de salto en la Sabana de Bogotá. Revista de Medicina Veterinaria Print versión Rev. Med. Vet.no.17. Bogotá Jan./June http://goo.gl/t9isAZ

Gutiérrez, JF. y Gonzales, C. (1998). Fisiología aplicada a la veterinaria y zootecnia. Centro de ediciones Universidad de Caldas. Manizales: Universidad de Caldas. P 180.

Hobo, S., Yoshida, K. and Yoshihara, T. (1998). Characteristics of Respiratory Function during Swimming Exercise in Thoroughbreds. Equine Research Institute. Japan Racing Association. 321-4 Tokami-cho, Utsunomiya-shi, Tochigi 320-0856.

Hottois, G. (2007). ¿Qué es la Bioética? Bogotá: Universidad el Bosque. 21 p.

Hoyos, J. (2016). Evaluación comparativa de un método de doma humanitaria y uno tradicional para el caballo de silla colombiano. Trabajo de grado para optar al título de magister. Maestría en sistemas sostenibles de producción. Tutor. Gloria Elena Estrada Cely. Florencia. Caquetá. Colombia. Universidad de la Amazonia. Facultad de ciencias Agropecuarias.

Hoyos, JF. (2020). El lomo del caballo (caballos pandos) segunda parte. https://bit.ly/2I1sGbU

Hoyos, JF., Franco, E., y Vargas, SA. (2020a). Natación en equinos, primera parte. https://bit.ly/2JtJxVD

Hoyos, JF., Franco, E., y Vargas, SA. (2020b). Natación en equinos, segunda parte. https://bit.ly/3ogkgNf

Patiño, JF., Gutiérrez, EB., Villamizar, D., y Carrascal, BLV. (2019). Aplicación del protocolo Welfare Quality® en criaderos equinos para determinar el grado de bienestar animal. Mundo FESC, 9(18), 24-30. https://dialnet.unirioja.es/servlet/articulo?codigo=7452804

Patiño-Quiroz, BE., Baldrich-Romero, NE., Caicedo-Robayo, JC., Ome-Peña, H., y Murillo-Rojas, JC. (2016). Prevalencia de anemia infecciosa equina en caballos de tracción en el municipio de Florencia (Caquetá). Revista Ciencia y Agricultura, 13(2), 39-45

Ramírez B, G. (2005). Manual de semiología clínica veterinaria. Editorial Universidad de Caldas. Ciencias agropecuarias. Manizales: Universidad de Caldas.

Taylor, S., y Bogdan, R. (2004). El proceso de la investigación científica. México: Limusa. 